\title{
Nonlinear $\sigma$ Model Treatment of Quantum Antiferromagnets in a Magnetic Field
}

\author{
B. Normand, Jordan Kyriakidis, and Daniel Loss \\ Departement für Physik und Astronomie, Universität Basel, Klingelbergstrasse 82, CH-4056 Basel, Switzerland.
}

(February 15, 2018)

\begin{abstract}
We present a theoretical analysis of the properties of low-dimensional quantum antiferromagnets in applied magnetic fields. In a nonlinear $\sigma$ model description, we use a spin stiffness analysis, a $1 / \mathrm{N}$ expansion, and a renormalization group approach to describe the broken-symmetry regimes of finite magnetization, and, in cases of most interest, a low-field regime where symmetry is restored by quantum fluctuations. We compute the magnetization, critical fields, spin correlation functions, and decay exponents accessible by nuclear magnetic resonance experiments. The model is relevant to many systems exhibiting Haldane physics, and provides good agreement with data for the two-chain spin ladder compound $\mathrm{CuHpCl}$.
\end{abstract}

PACS numbers: 75.10.Jm, 75.30.Cr, 75.40.Cx

\section{INTRODUCTION}

The importance of low-dimensional spin systems in revealing fundamental quantum mechanical properties has been recognized since Haldane's conjecturd concerning the effects of quantum fluctuations in integral- and halfintegral-spin antiferromagnetic (AF) chains. The key quantity is the topological term arising from the quantum spin phase, and similar considerations have since been extended to anisotropic and higher-order interactions, higher dimensions (planes), and coupled chains (spin ladders), largely driven by materials and experiment. Magnetic flux within a sample can alter the effective spin magnitude, and features visible in an applied field include spin gaps and magnetization plateaus.

Recent progress in metalloorganic synthesis has furnished new classes of low-dimensional antiferromagnets (AF) whose small exchange constants make their full magnetization response, including saturation, accessible to laboratory fields. The measured magnetization curves of some such materials, thought to be prototypical quantum magnets, in fact exhibit certain features which are rather classical. Examples on which experimental inter est has focused are the Haddane $(S=1)$ chain NENP, 3 the planar sygtem CFTD 4 and the two-chain spin ladder $\mathrm{CuHpCl}$. $\mathrm{CuHpCl}$, the best-characterized sample in recent literature.

We consider the quantum AF system in an external magnetic field using the nonlinear $\sigma$ model (NLsM). While this widely-applied treatment is in fact semiclassical, being truly valid only in the limit of large on-site spin $S$, it has in the past formed the basis for many fundamental deductions concerning the quantum limit of antiferromagnetic spin systems 9 . We will demonstrate here its validity in the case of all effectively integral-spin quantum systems in appreciable magnetic fields, and provide justification for this result in terms of suppression of quantum fluctuations by the field. Such systems display an ideal quantum phase transition, driven by the applied field, between a disordered regime with gapped spin exci- tions at low field and a quasi-long-range ordered regime with gapless excitations at higher field.

This model presents an ideal example of symmetry breaking in condensed matter systems. The $\mathrm{O}(3)$ symmetry of the spin Hamiltonian, and of its low-energy description in terms of the NLsM, is broken on a purely classical level. However, at low fields this symmetry is completely restored by strong quantum fluctuations, a well-known property of the NLsM, which has been used to illustrate the effect of asymptotic freedom (or "confinement of excitations"). 10 In contrast, quantum fluctuations may be suppressed by fields which are sufficiently strong but remain experimentally accessible, with the consequence that the full $\mathrm{O}(3)$ symmetry cannot be restored, and the spin system is reduced to the lower $\mathrm{XY}$ or $\mathrm{O}(2)$ symmetry. This XY symmetry, in the plane perpendicular to the applied field direction, remains sufficient that there can be no true long range order, but instead a quasilong-range order characterized by spin-spin correlation functions which decay in space-time with a power law form.

The outline of this paper is as follows. In Sec. II we derive the form of the NLsM in a magnetic field, using the geometry of the two-chain ladder system. With a view to experimental comparison we include magnetic saturation by applying a total-spin constraint. In Sec. III we analyze the spin stiffness of the model, which allows us to deduce the general behavior, correlation length, spin gap and critical field at zero temperature, and also the effects of finite temperature and system size. In Sec. IV we consider the $1 / N$ expansion, which is well suited for describing the low-field, disordered regime. Sec. V contains an extensive renormalization group (RG) study of the model over the entire field range. We derive the coupled RG equations for the $1+1$-dimensional $(1+1 \mathrm{~d})$ system and present their general solution. This allows us to show how the equations return the physics of symmetry-breaking and -restoration in the previous paragraph, and to discuss the issue of renormalization and gauge-invariance. We compute in Sec. VI the magnetization of the model using a high-field expansion, and in Sec. VII calculate spin 


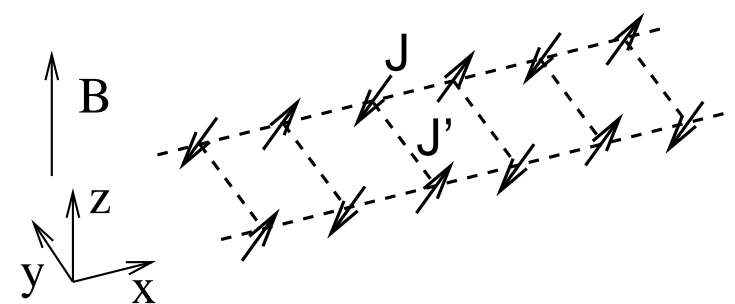

FIG. 1. Representation of two-chain ladder system and coordinate axes. $J=J_{x}$ is the intrachain and $J^{\prime}=2 J_{y}$ the interchain exchange coupling.

correlation functions for the low- and high-field regimes. In Sec. VIII we compare our results with experimental data from magnetization and nuclear magnetic resonance (NMR) spin-relaxation measurements. Sec. IX summarizes our conclusions, and discusses the variety of systems and physical problems to which the formalism is applicable.

\section{NONLINEAR $\sigma$ MODEL}

We consider the ladder geometry of the compound $\mathrm{CuHpCl} .5$ The Hamiltonian for the system in a magnetic field $\mathbf{b}=\tilde{g} \mu_{B} \mathbf{B}$ may be written as

$$
\hat{H}=\sum_{i ; m=1,2}\left(J \hat{\mathbf{S}}_{m, i} \cdot \hat{\mathbf{S}}_{m, i+1}+J^{\prime} \hat{\mathbf{S}}_{1, i} \cdot \hat{\mathbf{S}}_{2, i}+\mathbf{b} \cdot \hat{\mathbf{S}}_{m, i}\right)
$$

where $J$ is the superexchange interaction between spins in each chain and $J^{\prime}$ the interchain, or ladder "rung", interaction. We choose $N_{x}$-site chains with periodic boundary conditions in both directions, and the geometry shown in Fig. 1, such that $J_{x}=J, J_{y}=J^{\prime} / 2$, $\mathbf{B}=(0,0, B)$, and $\hat{\mathbf{S}}_{m, N_{x}+i}=\hat{\mathbf{S}}_{m, i}$. Following hestandard procedure in the derivation of the NLsM, 1114 the coherent-state representation of the spin $\hat{\mathbf{S}}_{m, i}$ is written as $S \boldsymbol{\Omega}_{m, i} \simeq S\left[(-1)^{i+m} \mathbf{n}_{m, i}+a \mathbf{l}_{m, i}\right]$, where $\mathbf{n}_{m, i}$ is a staggered spin (the Néel vector) with unit magnitude, and $\mathbf{l}_{m, i}$ describes the spin fluctuations perpendicular to $\mathbf{n}_{m, i}$. On proceeding to the continuum limit, the Hamiltonian is expressed in coherent-state representation as

$$
H=\int d \mathbf{r}\left(\frac{1}{2} S^{2} \sum_{j=x, y} J_{j}\left[\left(\partial_{j} \mathbf{n}\right)^{2}+4 \mathbf{l}^{2}\right]-\frac{S}{a} \mathbf{l} \cdot \mathbf{b}\right) .
$$

The full Euclidean action $\mathcal{S}_{E}=\mathcal{S}_{W Z}+\int_{0}^{\beta} d \tau H$ in space $\mathbf{r}=(x, y)$ and inverse temperature $\tau\left(\beta=1 / k_{B} T\right)$ contains a Berry-phase, or Wess-Zumino, term whose origin lies in the solid angle subtended by the fluctuating quantum spin phases:
$\mathcal{S}_{W Z}=i \frac{S}{a^{2}} \int d \tau d \mathbf{r}[\dot{\phi}-a \mathbf{l} \cdot(\mathbf{n} \wedge \dot{\mathbf{n}})]+4 \pi i S\left(P_{1}+P_{2}\right)$

Here, $\phi$ is the azimuthal angle, and $P_{1}=P_{2}=$ $(1 / 4 \pi) \int d \tau d x(\mathbf{n} \wedge \dot{\mathbf{n}}) \cdot \partial_{x} \mathbf{n}$ are topological terms which give the Pontryagin index on each chain when $\partial_{y} \mathbf{n}=0$, as is the case in a ladder of only two chains. 15 The last term in Eq. (2.3) is therefore $i\left(4 \pi P_{1}\right) 2 S$, demonstrating that the system will have integral-spin characteristics for any valun of $S$, and the topological term may thus be ignored.16.17

Integrating out the fluctuations $\mathbf{l}$ about the staggered spin configuration of $\mathbf{n}$, subject to the orthogapality constraint $\mathbf{n} \cdot \mathbf{l}=0$, yields the classical solution 14.18

$$
\mathbf{l}_{\mathrm{cl}}=\frac{1}{4 a S \bar{J}}\{i(\mathbf{n} \wedge \dot{\mathbf{n}})+[\mathbf{b}-\mathbf{n}(\mathbf{b} \cdot \mathbf{n})]\},
$$

where $\bar{J}=J_{x}+J_{y}$. Finally, the corresponding action for the quasi-one-dimensional (1D) ladder system, in $1+1$ Euclidean dimensions denoted by $\mu$, is

$$
\begin{aligned}
\mathcal{S}_{E}=\frac{1}{2 g} \int d \tau d x\left\{\left(\partial_{\mu} \mathbf{n}\right)^{2}-\right. & {\left[\mathbf{b}^{2}-(\mathbf{n} \cdot \mathbf{b})^{2}\right] } \\
+ & 2 i \mathbf{b} \cdot(\mathbf{n} \wedge \dot{\mathbf{n}})\},
\end{aligned}
$$

where $g=\left(2 / N_{y} S\right) \sqrt{\bar{J} / J_{x}}$ is the bare coupling constant, and the integral over $\tau$ is from zero to $L_{T}=c \beta$, with $c=2 S a \sqrt{J_{x} \bar{J}}$ the effective spin-wave velocity. (We use $\hbar=1$ throughout.) Summation over $\mu$ is implied in the square. We have left explicit the number of chains $N_{y}$ in the ladder. For $\mathrm{CuHpCl}$ we have that $J_{y}>J_{x}$ and $N_{y}=2$, whence the rung coupling may be treated as effectively rigid. The form of the NLsM in an external field given by Eq. (2.5) has been derived previously, 11 and its implications considered in the low-field limit. 18 We will examine in this work its properties at all field strengths.

The action (2.5) may be cast in a physically more transparent form by expressing the staggered spin in spherical coordinates, $\mathbf{n}=(\sin \theta \cos \phi, \sin \theta \sin \phi, \cos \theta)$. From the resulting Lagrangean density

$$
\mathcal{L}_{\mathrm{E}}=\frac{1}{2 g}\left\{\left[(\dot{\phi}+i b)^{2}+\left(\partial_{x} \phi\right)^{2}\right] \sin ^{2} \theta+\left(\partial_{\mu} \theta\right)^{2}\right\}
$$

one observes that the field induces a hard-axis anisotropy through the term $(\mathbf{n} \cdot \mathbf{b})^{2}$, which makes the spins tend to align with the Néel vector $\mathbf{n}$ in the plane normal to b. For strong fields, deviation of $\mathbf{n}$ from this plane is small, and expansion to quadratic order in $\vartheta=\theta-\pi / 2$ is valid. Taking the field along the $z$-axis, we find that the Lagrangean

$$
\begin{aligned}
\mathcal{L}_{\mathrm{E}}=\frac{1}{2 g} & \left\{\left[(\dot{\phi}+i b)^{2}+\left(\partial_{x} \phi\right)^{2}\right]\right. \\
& \left.-\vartheta\left[\partial_{\mu}^{2}+(\dot{\phi}+i b)^{2}\right] \vartheta\right\}
\end{aligned}
$$


separates into contributions from in-plane and out-ofplane fluctuations in the high-field regime, $|b|>\dot{\phi} \equiv \omega$. We will use Eq. (2.7), and return to the condition for its validity, below.

Finally, for a real magnetic system with small spin quantum number $S$, application of a sufficiently large magnetic field will cause total spin alignment, or saturation, with a maximum magnetization given by $M_{s}=$ $\tilde{g} \mu_{B} S N_{s}$, where $N_{s}\left(=N_{x} N_{y}\right.$ here $)$ is the total number of sites in the system. This effect is not contained in the NLsM (Eq. (2.5)) discussed above, where $S$ is required to be large (semi-classical). Furthermore a saturation of the spin would correspond to large $|\mathbf{l}|$ and small $|\mathbf{n}|$, rendering invalid the expansion of $\mathbf{S}$ (below Eq. (2.1)). We will include saturation by placing a limit, corresponding to the desired experimental spin magnitude, on the spin component $\left(S_{z}\right)$ projected along the field. By this technique, the full spin vector remains primarily in the plane, justifying the treatment of the uniform spin component |l $\mid$ as a small variable.

The succeeding experimental comparison (Sec. VIII) will be simplified by treating $M_{s}$ as a simple cutoff, but we describe here briefly this systematic inclusion of saturation in the model by the application of a constraint on the total spin. In addition to the constraints $\mathbf{n} \cdot \mathbf{l}=0$ on the uniform and staggered spin components, and $\mathbf{n}^{2}=1$ on the magnitude of the staggered spin, we introduce a further constraint enforcing conservation of spin. For a field applied along the $z$ axis, the conserved quantity is the $z$-component of the total spin $S^{z} \equiv\left\langle\{\boldsymbol{\Omega}\}\left|\widehat{S}^{z}\right|\{\boldsymbol{\Omega}\}\right\rangle \rightarrow S \int d x l^{z}$, where the continuum limit is taken in the final step. While the previous constraints are purely local, in that they must be enforced at each spacetime point, the spin constraint is local in time but global in space. All of the constraints are treated by inserting (functional) delta functions into the measure of the propagator, in the form

$$
\begin{aligned}
& \left\langle\{\boldsymbol{\Omega}\}_{b}\left|e^{-\beta H}\right|\{\boldsymbol{\Omega}\}_{a}\right\rangle \\
& \quad=\int \mathcal{D} \mathbf{n}(x, \tau) \int \mathcal{D} \mathbf{l}(x, \tau) e^{-f_{\mathrm{C}}[\mathbf{n}, \mathbf{l}]} e^{-\mathcal{S}_{E}[\mathbf{n}, \mathbf{l}]},
\end{aligned}
$$

where the function $f_{\mathrm{C}}[\mathbf{n}, \mathbf{l}]$ specifies the constraints using Lagrange multipliers. Integrating over the fluctuating spin field $\mathbf{l}$, which is now constrained, and over all the Lagrange multipliers, one obtains the Euclidean action

$$
\begin{aligned}
\mathcal{S}[\mathbf{n}]= & \mathcal{S}_{W Z}+\frac{1}{2 g} \int d \tau d x\left(\partial_{\mu} \mathbf{n}\right)^{2} \\
& -\frac{1}{2 g} \int d \tau \frac{\left(D[\mathbf{n}]+2 i N_{s} \sigma\right)^{2}}{A[\mathbf{n}]}-S N_{s} \beta b \sigma
\end{aligned}
$$

where $D[\mathbf{n}]=\int d x(\mathbf{n} \wedge \dot{\mathbf{n}})_{z}$ and $A[\mathbf{n}]=\int d x\left(1-n_{z}^{2}\right)$, and we have introduced the scaled spin $\sigma=S_{z} /\left(S N_{s}\right),-1 \leq$ $\sigma \leq 1$. In this action the field $b$ is coupled only linearly to the spin $\sigma$, as in ferromagnetic systems, and in contrast to Eq. (2.5), where it appears quadratically. This linear coupling is a direct consequence of the spin constraint,

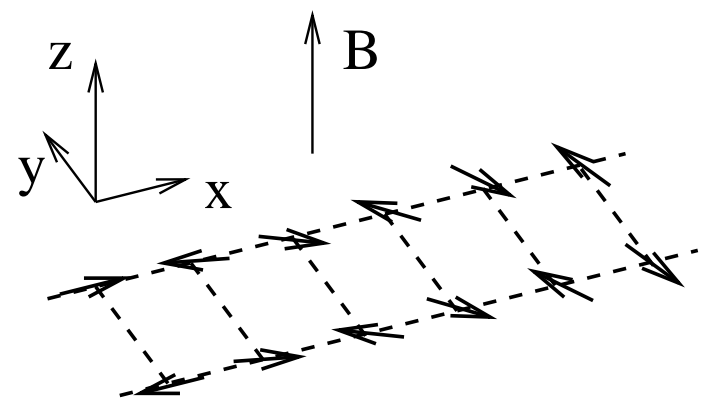

FIG. 2. Ladder system with twisted staggered moment.

and one may recover the previous action (Eq. (2.5)) by allowing $\sigma$ to fluctuate in time, relaxing the condition $|\sigma| \leq 1$, and integrating over $\sigma(\tau)$. Evaluation of the partition function with saturation, requires summing the constrained action (Eq. (2.9)) over all degrees of freedom, which include the allowed values of $\left|S_{z}\right| \leq N_{s} S$. This procedure limits $M$ to $M_{s}$ at large fields, and provides a consistent description of a second phase transition, to saturation, which occurs at high fields. In Secs. III-V we will concentrate on the symmetry-breaking quantum phase transition (Sec. I), which occurs at a lower field on the order of the zero-field gap (below), and so the discussion there will not involve further consideration of the spin constraint.

\section{SPIN STIFFNESS}

To gain initial insight into the effect of the magnetic field, we consider the spin stiffness of the ladder system using the method of Ref. 19.20 We take (Fig. 2) the staggered spin configuration to be subject to a twist $\psi$ in the plane, $\mathbf{n}(\tau, 0)=(1,0,0)$ and $\mathbf{n}(\tau, L)=(\cos \psi, \sin \psi, 0)$. This is conveniently represented by $\mathbf{n}=\mathcal{R}(x) \boldsymbol{\sigma}$, where $\mathcal{R}$ is a matrix for rotation by $\psi x / L$ about the $z$ axis, and $\boldsymbol{\sigma}=\boldsymbol{\sigma}(\tau, x)$ a 3 -component vector with value $(1,0,0)$ at the spatial endpoints. The cross-term in $\left(\partial_{\mu} \mathbf{n}\right)^{2}$ and the third term in Eq. (2.5) are total derivatives in $x$ and $\tau$ respectively, and will be discarded for the present purposes. This is equivalent to considering only paths with no winding in Euclidean space, and gives the action the form

$$
\mathcal{S}_{E}=\frac{1}{2 g} \int d \tau d x\left\{\left(\partial_{\mu} \boldsymbol{\sigma}\right)^{2}+\left(\frac{\psi^{2}}{L^{2}}-\frac{b^{2}}{c^{2}}\right)\left(1-\sigma_{3}^{2}\right)\right\} .
$$

To 1-loop order in $g$, the spin stiffness is given by

$$
\begin{aligned}
\rho_{s} & =\left.\frac{1}{2} c L \frac{\partial^{2} F}{\partial \psi^{2}}\right|_{\psi=0} \\
& =\left.\frac{c L}{2 L_{T}} \frac{\partial^{2}}{\partial \psi^{2}}\right|_{\psi=0}\left\{\frac{L L_{T}}{2 g}\left[\frac{\psi^{2}}{L^{2}}-\frac{b^{2}}{c^{2}}\right]\right.
\end{aligned}
$$




$$
\begin{gathered}
\left.+\frac{1}{2} \operatorname{Tr} \ln \left[\mathbf{k}^{2}-\frac{\psi^{2}}{L^{2}}+\frac{b^{2}}{c^{2}}\right]\right\} \\
=\rho_{s}^{0}\left[1-\frac{g}{L L_{T}} \sum_{\mathbf{k}} \frac{1}{\mathbf{k}^{2}+(b / c)^{2}}\right],
\end{gathered}
$$

where $\rho_{s}^{0}=c / 2 g$ is the classical (bare) value, and the sum includes both quantum and thermal (through the finite "length" $L_{T}$ ) corrections to first order in $g$.

We consider first the low-temperature, or "quantum" regime $L_{T} \gg L$. We evaluate the summation between the spatial limits $\pi / L$ and $\pi / a$ (this change to open boundary conditions does not affect the result), and introduce the "magnetic length"

$$
L_{m}=\pi c / b
$$

to obtain the expression

$$
\rho_{s}=\rho_{s}^{0}\left[1+\frac{g}{2 \pi} \ln \left(\frac{a / L+\sqrt{(a / L)^{2}+\left(a / L_{m}\right)^{2}}}{\left(1+\sqrt{\left.1+\left(a / L_{m}\right)^{2}\right)}\right.}\right)\right] .
$$

The system length $L$ in Eq. (3.4) may be substituted by a correlation length $\xi$, beyond which segments of the ladder behave independently, and $\xi$ then computed from the condition $\rho_{s}=0$. We emphasize that $\rho_{s}$ is to be considered as a local stiffness, meaningful only on length scales $L \leq \xi$. In the zero-field limit $\left(L_{m} \rightarrow \infty\right)$ one obtains the result $\xi_{0}=A a e^{2 \pi / g}=A a e^{\alpha \pi S}$, where $\alpha=\sqrt{J_{x} / \bar{J}}$ intra duces a dependence on the ladder coupling constants, 17 and $A$ is a nonuniversal constant of order unity which depends on the integration cutoff. The general solution can be expressed as

$$
\begin{aligned}
\xi(B) & =2 L_{m}^{*} /\left[1-\left(L_{m}^{*} / L_{m}\right)^{2}\right] \\
& \equiv \xi_{0} /\left[1-\left(B / B^{*}\right)^{2}\right],
\end{aligned}
$$

from which we see that the correlation length $\xi(B)$ diverges at a critical field $B^{*}$, where $L_{m}^{*}=a \sinh (2 \pi / g)$. For fields $B<B^{*}$, the system has only shortrange correlations, and the finite correlation length may be written as $\xi(B)=a e^{\alpha \pi \tilde{S}}$, where $\tilde{S}=$ $S\left[1-(g / 2 \pi) \ln \left(1-\left(L_{m}^{*} / L_{m}\right)^{2}\right)\right]$ is a growing value of the effective spin. There is no spontaneous breaking of the $O(3)$ spin symmetry. For $B>B^{*}$, the field en forces a long-ranged correlation throughout the system, 21 and it is most convenient to write the spin stiffness as $\rho_{s}=\rho_{s}^{0}\left[1-(g / 2 \pi) \ln \left(1+L_{m} / a\right)\right]$, a quantity which recovers the bare value $\rho_{s}^{0}$ as $B \rightarrow \infty$. Finally, at the transition $B^{*}$, it is clear from Eq. (3.5) that the diverging correlation length corresponds to the closing of a gap $\Delta \sim \xi^{-1}$ to spin excitations with the form

$$
\Delta \propto 1-\left(B / B^{*}\right)^{2} .
$$

This situation is summarized in Fig. 3. While $\Delta$ is the gap to all fluctuations in the low-field regime where $O(3)$

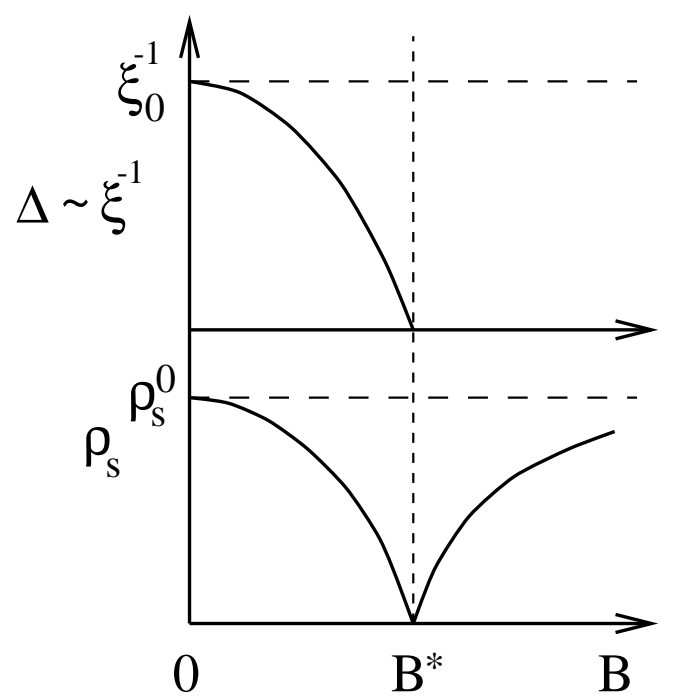

FIG. 3. Schematic behavior of spin stiffness and correlation length, or spin gap, with applied field.

spin symmetry is maintained, we note that at high fields not all the modes are gapless. In this regime (strictly $B \gg B^{*}$ ) the symmetry is lowered to $O(2)$, and while inplane $(\phi)$ fluctuations (Eq. (2.7)) are massless, excitation modes $(\vartheta)$ in the direction of the field, or out of the plane it enforces, are gapped with "mass" $b$.

We may further employ the spin stiffness as a means of characterizing the behavior of a system of finite size $L$, and at finite temperatures, for different values of the magnetic field. This is essentially a matter of comparing the corresponding length scales $L, L_{T}$ and $L_{m} 19$. We present results for the cases of i) weak and ii) strong fields in the following three regimes: a) quantum, i) $L_{m}, L_{T} \gg$ $L$ and ii) $L_{T} \gg L \gg L_{m}$, b) classical renormalized, i) $L_{m}, L \gg L_{T}$ and ii) $L \gg L_{T} \gg L_{m}$, and c) classical, i) $L_{m}, L \gg a \gg L_{T}$ and ii) $L \gg L_{m}, a \gg L_{T}$. Following Ref. 19 we quote the stiffness in dimensionless units of $\rho_{s} / c=(1 / 2 g)\left(\rho_{s} / \rho_{s}^{0}\right)$. For weak fields we find

$$
\frac{\rho_{s}}{c}= \begin{cases}\frac{1}{4 \pi} \ln \left[\frac{\xi_{o}}{2}\left(\frac{1}{L}+\sqrt{\frac{1}{L^{2}}+\frac{1}{L_{m}^{2}}}\right)\right], & L_{m}, L_{T} \gg L \\ \left(\xi_{B}(T)-\min \left[L, L_{m}\right]\right) / 12 L_{T}, & L_{m}, L \gg L_{T} \\ \left(\xi_{\mathrm{cl}}-L\right) / 12 L_{T}, & L_{m}, L \gg a \gg L_{T} .\end{cases}
$$

$\xi_{0}$ denotes the quantum correlation length defined above, $\xi_{\mathrm{cl}}=6 L_{T} / g$ is the classical correlation length, and

$$
\xi_{B}(T)=\frac{3 L_{T}}{\pi}\left[\ln \left(C \frac{\xi_{0}}{2 L_{T}} \sqrt{1+\sqrt{1+\left(L_{T} / L_{m}\right)^{2}}}\right)\right],
$$

with $C \sim O(1)$ another nonuniversal constant, contains the renormalizing effect of quantum fluctuations on $\xi_{\mathrm{cl}}$. These results are all directly analogous to the zero-field case, 19 with the field entering as a quadratic correction where relevant. In a strong applied field (ii), 


$$
\frac{\rho_{s}}{c}= \begin{cases}\frac{1}{4 \pi} \ln \left[\frac{\xi_{o}\left(\sqrt{1+\left(L_{m} / L\right)^{2}}\right)}{L_{m}+\sqrt{a^{2}+L_{m}^{2}}}\right], & L_{T} \gg L \gg L_{m} \\ \left(\xi_{T}(B)-L_{m}\right) / 12 L_{T}, & L \gg L_{T} \gg L_{m} \\ \left(\xi_{\mathrm{cl}}-L_{m}\right) / 12 L_{T}, & L \gg L_{m}, a \gg L_{T},\end{cases}
$$

where now the renormalized classical correlation length has the field-dominated form

$\xi_{T}(B)=\frac{3 L_{T}}{\pi}\left[\ln \left(C \frac{\xi_{0}}{L_{m}} \sqrt{\sqrt{1+\left(L_{m} / L_{T}\right)^{2}}+L_{m} / L_{T}}\right)\right]$.

The general characteristic of all the above results is a logarithmic dependence of the spin stiffness on the length scales of system size, temperature, and field where quantum fluctuations are important, turning to a power-law dependence in the classical regime. In the high-field situation the scale $L_{m}$ of the field replaces $L_{T}$ as the determining parameter (cf. Eqs. (3.7, 3.9)). Equivalent results for finite systems may also be deduced from the $1 / N$ expansion, which we consider in the following section.

We will later compute the magnetization $M=$ $\partial F /\left.\partial B\right|_{\psi=0}$, both in the thermodynamic limit and for systems of finite size, from the same type of free energy as in Eq. (3.2). This undertaking is simplified in the light of insight gained from $1 / N$ and $\mathrm{RG}$ analyses, and is thus deferred to Sec. VI. At this point we note only that the magnetization calculated in this framework is not zero in the low-field regime, as would be required of a system with a spin gap and no broken symmetry. This is a consequence of the approximation, implicit in Eq. (2.7) and in deriving the free energy $F(b)$ (Eq. (3.2)), that the field $b$ be large on the scale of $|\dot{\phi}|$. We may nevertheless conclude that analysis of the spin stiffness itself is qualitatively revealing of many properties of the system.

\section{1/N EXPANSION}

To address the situation in the weak-field regime, we adopt instead a $1 / N$ expansion, 10 which is expected to be appropriate in describing spin-gap phases. Here the staggered spin $\mathbf{n}$ is assumed to exist in an $N$-dimensional spin space, in which only the direction $n_{z}$ is selected by the magnetic field, and a controlled expansion may in principle be performed in the small quantity $1 / N$ to compute fluctuation corrections to the saddle-point solution. The relevant parts of the action are (cf. Eq. (3.1))

$$
\mathcal{S}_{E}=\frac{1}{2 g} \int d \tau d x\left[\left(\partial_{\mu} \mathbf{n}\right)^{2}-\bar{b}^{2}\left(1-n_{z}^{2}\right)-i \lambda\left(\mathbf{n}^{2}-1\right)\right],
$$

where $\bar{b}$ denotes $b / c$, and the constraint that the spin $\mathbf{n}$ have unit magnitude is made explicit with the Lagrange multiplier $i \lambda$. Integration over $\mathbf{n}$ and taking the functional derivative of the resultant effective action with respect to $\lambda$ yield an equation for the saddle-point value of the Lagrange multiplier,

$$
\frac{1}{g}=(N-1) \sum_{\mathbf{k}} \frac{1}{\mathbf{k}^{2}+i \lambda}+\sum_{\mathbf{k}} \frac{1}{\mathbf{k}^{2}+i \lambda+\bar{b}^{2}},
$$

in which the $B$-field term is found to appear only at $O(1 / N)$. $i \lambda$ appears as a mass, or cutoff term in momentum integrations, and is thus an upper length scale for cooperative processes in the system, or simply a correlation length (inverse excitation gap). Thus by writing the saddle-point solution as $i \lambda=c^{2} \pi^{2} / \xi(B)^{2}$, and carrying out the summation at low $T$ from 0 to $\pi / a$, this correlation length is determined from

$$
\begin{aligned}
1=-\frac{g}{2 \pi} \ln & {\left[\left(\frac{a / \xi}{1+\sqrt{1+(a / \xi)^{2}}}\right)^{N-1}\right.} \\
\times & \left.\left(\frac{\sqrt{(a / \xi)^{2}+\left(a / L_{m}\right)^{2}}}{1+\sqrt{1+(a / \xi)^{2}+\left(a / L_{m}\right)^{2}}}\right)\right],
\end{aligned}
$$

which should be compared with $\rho_{s}=0$ emerging from Eq. (3.4).

In the weak-field regime, $\xi \ll L_{m}$, one finds

$$
\xi \simeq(1 / 2) a e^{2 \pi / N g}\left[1+\left(a^{2} / 8 N L_{m}^{2}\right) e^{4 \pi / g}\right] .
$$

This is an $O(1 / N)$ correction to the previous result, but there is also a difference of a power of $1 / N$ in the exponent. Taking the field derivative, the result $\partial_{b}(c \pi / \xi)^{2}=-2 b / N$ ensures that the magnetization contribution from the $\mathbf{k}$ summation terms in $F$ are identically zero to $O(1 / N)$. At the same order, the field derivative of the $\mathbf{k}$-independent term $b^{2}+c^{2} \pi^{2} / \xi^{2}$ is $2 b(1-1 / N)$. The behavior required of a gapped system is obtained in the weak-field regime on making the well-known identification, deduced by comparison with exact RG results (see Ref. 10 and below) $N \rightarrow N-2$. Returning to the physical situation of the $O(3)$ magnet, the magnetization is zero at all fields $B<B^{*}$. The saddle-point solution for $\xi(B)(4.3)$ becomes precisely that deduced from Eq. (3.4) (up to a negligible term retained from a lower cutoff), with the same field $B^{*}$ for divergence of $\xi$. For the $O(3)$ case we thus deduce the expected gapped state, with no breaking of spin rotational symmetry.

For completeness, we note the strong-field results of the $1 / N$ treatment. When the magnetic length is small $\left(L_{m} \ll \xi\right)$, the correlation length $\xi \simeq$ $(1 / 2) a e^{2 \pi / g(N-1)}\left(a / L_{m}\right)^{1 /(N-1)}$ has a direct field dependence. The magnetization is given to leading order by $M \simeq-C+2 b L_{m}^{2} /\left[(N-1) \xi^{2}\right]$. The quantity appearing in these expressions is not $N$ but $N-1$, indicating again the lowering of symmetry in the field direction above a finite critical field $B^{*}$. However, these high-field results are not meaningful in the $O(3)$ case. 
In summary, the spin stiffness and $1 / N$ techniques taken together yield a consistent picture at both weak and strong magnetic fields. However, while the Ansatz $N \rightarrow N-2$ has been applied previously 10 and leads to consistency between the results of this and the previous section, it cannot be taken to be entirely satisfactory. For this reason we proceed by applying the $\mathrm{RG}$ technique to the model of Eq. (2.5).

\section{RENORMALIZATION GROUP}

We consider next a renormalization-group (RG) study of the NLsM in an applied field. This approach yields meaningful results over the full parameter range. We adopt a standard Wilson momentum-shell treatment, further details of which are presented in App. 1. In brief, the action in the form

$$
\mathcal{S}_{E}=\frac{1}{2 g} \int_{0}^{L_{T}} d \tau \int_{0}^{L} d x\left[\left(\partial_{\mu} \mathbf{n}\right)^{2}-\bar{b}^{2}\left(1-n_{z}^{2}\right)+4 i \bar{b} n_{x} \dot{n}_{y}\right]
$$

may be reexpressed in terms of variables $\phi$, representing in-plane fluctuations of $\mathbf{n}$, and $\sqrt{g} \sigma_{z}=n_{z}$, representing out-of-plane fluctuations. The latter is chosen to facilitate a perturbative expansion in $g$, which yields

$$
\mathcal{L}_{E}=\frac{1}{2 g}\left(A^{\prime}-\bar{b}^{2}\right)-\frac{1}{2} \sigma_{z}\left(-\partial_{\mu}^{2}+\bar{b}^{2}-A^{\prime}\right) \sigma_{z}+O(g) .
$$

$A^{\prime}=\left(\partial_{\mu} \phi\right)^{2}+2 i \bar{b} \dot{\phi}$ denotes in-plane fluctuation terms, which, because $\phi(\tau, x)$ is assumed to vary slowly, can be taken to be a small constant (no fast Fourier modes) in the momentum shell $\gamma \Lambda<|\mathbf{k}|<\Lambda(\gamma \rightarrow 1)$ around the finite upper cutoff $\Lambda=\pi / a$ set by the lattice length scale. By expanding in $A^{\prime}$, the form of Eq. (5.2) is recovered, but with new coefficients $g\left(a^{\prime}\right)$ and $\bar{b}\left(a^{\prime}\right)^{2}$ given at 1-loop order by

$$
\begin{aligned}
\frac{1}{g\left(a^{\prime}\right)} & =\frac{1}{g_{0}}-\operatorname{Tr}^{\prime} \frac{1}{-\partial_{\mu}^{2}+\bar{b}^{2}} \\
\bar{b}\left(a^{\prime}\right)^{2} & =\bar{b}_{0}^{2}-g_{0} \operatorname{Tr}^{\prime} \ln \left(-\partial_{\mu}^{2}+\bar{b}_{0}^{2}\right) .
\end{aligned}
$$

The integrals represented by the partial traces $\left(\operatorname{Tr}^{\prime}\right)$ are performed over an isotropic momentum shell in $1+1 \mathrm{~d}$, with $\Lambda_{T}=\Lambda$. Alterations arising from considering instead a rectangular momentum shell, with separate integration over space and inverse temperature, would affect only non-universal prefactors.

Inspection of the equation (5.3) for renormalization of the coupling constant $g$ indicates already the effect of the field. Performing the momentum-shell integral yields

$$
\frac{g_{0}}{g\left(a^{\prime}\right)}=1-\frac{g_{0}}{4 \pi} \ln \frac{1+\left(a / L_{m}\right)^{2}}{\left(a / a^{\prime}\right)^{2}+\left(a / L_{m}\right)^{2}}
$$

for $a \leq a^{\prime} \leq \mathcal{L}=\min \left[L, L_{T}\right]$, assuming (see below) that the flow of $L_{m}$ is at most weak. For weak fields $L_{m} \gg \mathcal{L}$ we see that $g_{0} / g(\mathcal{L})=1-\left(g_{0} / 2 \pi\right) \ln (\mathcal{L} / a)$, i.e. $g$ is renormalized by the full effects of quantum fluctuations. For strong fields $\mathcal{L} \gg L_{m} \gg a$, we have $g_{0} / g(\mathcal{L})=1-\left(g_{0} / 2 \pi\right) \ln \left(L_{m} / a\right)$, and the coupling constant $g$ is independent of the system length scale $\mathcal{L}$. In this case renormalization is weak, and for very strong fields $L_{m} \ll a$ it vanishes $\left(g_{0} / g(\mathcal{L})=1\right)$, demonstrating the suppression of quantum fluctuation effects by high fields.

Following the standard RG procedure, we define the flow parameter $l=\ln \left(a^{\prime} / a\right)$, whence $d l=d a / a$. Taking the derivative with respect to $l$ of Eqs. (5.3,5.4), we obtain the differential form of the coupled $\mathrm{RG}$ equations

$$
\begin{aligned}
\frac{d g}{d l} & =\frac{g^{2}}{2 \pi} \frac{1}{1+\bar{\beta}^{2}}, \\
\frac{d \bar{\beta}^{2}}{d l} & =2 \bar{\beta}^{2}-\frac{g}{2 \pi} \ln \left(1+\bar{\beta}^{2}\right) .
\end{aligned}
$$

These represent an extension of the usual RG equations to include a magnetic field $B$, contained in $\bar{\beta}=a^{\prime} \bar{b}\left(a^{\prime}\right)(=$ $\left.a^{\prime} b\left(a^{\prime}\right) / c\right)$. Eq. (5.6) is the conventional " $\beta$-equation", the terminology given to the renormalization of the coupling constant, but with an additional field term in the denominator. Clearly, a strong field restricts flow to the strong-coupling (disordered) limit, and, as observed in the previous paragraph, acts to suppress quantum fluctuation effects. This points to a "deconfinement of excitations" at suitably high field. Eq. (5.7) expresses the renormalization of the magnetic field term $b=\tilde{g} \mu_{B} B$ with dynamical exponent $z=1$ (from the first term, which arises purely from the presence of $a^{\prime}$ in the quantity $\beta$ ), but with additional, logarithmic suppression of this flow at strong field and coupling (second term).

At this point some commentary is in order regarding the above RG equations. The NLsM in the presence of a magnetic field may be expressed in the gauge theoretic form 22,23

$$
\mathcal{L}=\frac{c}{2 g}\left[\frac{1}{c^{2}}\left(\partial_{\tau} n_{\alpha}+\frac{i \tilde{g} \mu_{B}}{\hbar} \epsilon_{\alpha \beta \gamma} B_{\beta} n_{\gamma}\right)^{2}+\left(\partial_{x} n_{\alpha}\right)^{2}\right]
$$

with the field term $\tilde{g} \mu_{B} B / c$ in the rqle of a gauge potential. From this it has been argued 23 that the field term should scale only with temperature (the $z=1$ behavior of the first term in Eq. (5.7)), and cannot be renormalized separately, for example by interactions, as this would violate the gauge invariance. This scaling hypothesis stands in contrast to the result (Eq. (5.7)) of the Wilson momentum-shell RG approach taken above, where the second term indeed gives a field renormalization, albeit one which is logarithmically weak. However, the present situation is not unique: similar breakdown of this scaling occurs in other cases, particular examples being the 


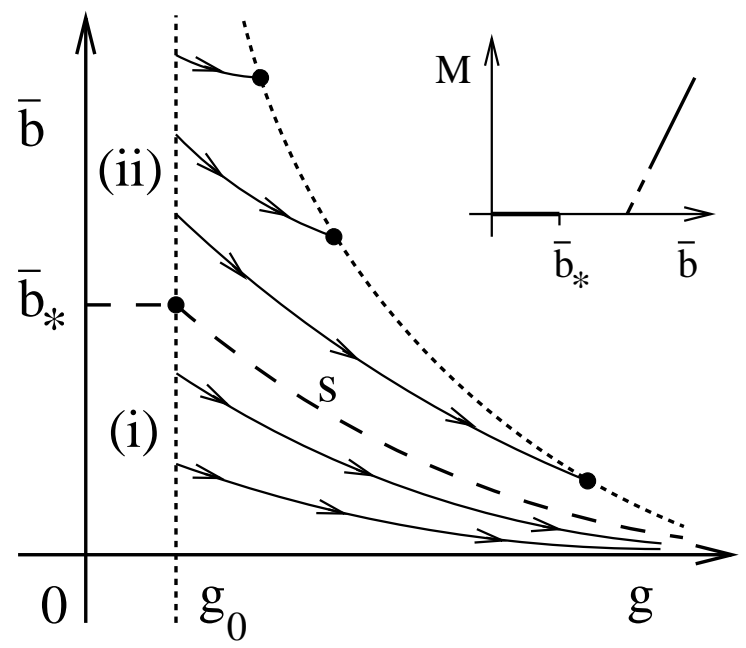

FIG. 4. RG flow diagram for $g$ and $\bar{b}$. Strong- and weak-coupling regimes are separated by separatrix $s$.

Fermi liquid and the Luttinger liquid, also considered in Ref. 23, where there is a temperature-independent renormalization of the field term 24.25 We note also that on taking into account magnetic saturation (Sec. II) in the present model, additional field terms would arise which preclude the use of a gauge-invariance argument. Finally, the qualitative features of the discussion are not affected by the logarithmic term in Eq. (5.7), because its field renormalization is weak.

In analyzing the content of the RG equations (5.6,5.7), we concentrate first on the fixed points, in order to obtain a qualitative picture of the RG flow diagram. Seeking a fixed point by a weak-field expansion around $\bar{\beta}_{*}=0$, we find

$$
\frac{d g}{d l} \simeq \frac{g^{2}}{2 \pi}, \quad \frac{d \ln \bar{\beta}^{2}}{d l}=2-\frac{d \ln g}{d l},
$$

which may be solved to yield

$$
\frac{g_{0}}{g}=1-\frac{g_{0}}{2 \pi} l, \quad \bar{\beta}=\bar{\beta}_{0} e^{l}\left(1-\frac{g_{0}}{2 \pi} l\right)^{1 / 2} .
$$

The fixed point $\left(g_{*}, \bar{\beta}_{*}\right)=(\infty, 0)$ is evidently stable if the flow is stopped at $l_{*}=2 \pi / g_{0}$. The system will flow to this strong-coupling regime if the starting value $\bar{\beta}_{0}$ is sufficiently small. The length scale $\mathcal{L}_{*}=a e^{l_{*}}$ at which the flow stops may be compared with the spatial and thermal dimensions $\left(L, L_{T}\right)$ of the system to calculate directly the effects of finite size and temperature ( $c f$. Sec. III). At strong fields $(\bar{\beta} \rightarrow \infty), d g / d l=0$, or $g=g_{0}$, and similarly $d \ln \bar{\beta}^{2}=2 d l$, from which it follows that $\bar{b}(l)=\bar{b}_{0}$, i.e. in this regime neither the coupling nor the field is renormalized.

The RG equations (5.6.5.7) may be solved numerically in the form

$$
\frac{\partial x}{\partial l}=\frac{-1}{l\left(1+l^{2} y^{2}\right)}, \quad \frac{\partial y}{\partial l}=\frac{-1}{2 x y l^{3}} \ln \left(1+l^{2} y^{2}\right),
$$

where $x=2 \pi / g$ is the inverse coupling constant and $y=a \bar{b}$ corresponds to the field variable $b / c$ only. Solution leads to the flow diagram in Fig. 4, which has the following interpretation. The regime (i) of weak initial $B$-field is the strong-coupling phase, with confinement of (gapped) excitations. Here, the assumption (underlying the perturbative RG treatment) of small $g$ becomes inconsistent, but one may still deduce the critical length scale $\mathcal{L}_{*}$, and that the magnetization $M=0$. In this region $O(3)$-symmetry is restored by quantum fluctuations, which may thereby be considered as "screening" the magnetic field. In contrast, the regime (ii) of strong initial $B$-field corresponds to weak coupling, where $g$ and $\bar{b}$ are only weakly renormalized. Here, the excitations are deconfined on a length scale $\bar{\xi}(B)$ whose flow is governed by $B$. In this region, quantum fluctuations are suppressed by the magnetic field, and the broken $O(3)$ symmetry cannot be restored.

The properties of the broken- and unbroken-symmetry phases may be further contrasted by considering the correlation length $\xi$ in each regime. This is a physical quantity and so does not change under the RG flow, whence $d \xi / d a^{\prime}=0$ and

$$
\frac{\partial g}{\partial l} \frac{\partial \xi}{\partial g}+\frac{\partial \bar{\beta}^{2}}{\partial l} \frac{\partial \xi}{\partial \bar{\beta}^{2}}+\xi=0
$$

At low fields, we find that $-\partial \xi / \xi \simeq 2 \pi \partial g / g$, which has the solution $\xi_{0}=\xi e^{2 \pi\left(1 / g_{0}-1 / g\right)}$. If one assumes that $\xi \rightarrow a$ (the bare lattice constant), as the system flows to the strong-coupling limit $\left(1 / g_{0} \rightarrow 0\right)$, then $\xi_{0}=a e^{2 \pi / g_{0}}$ returns the same, finite physical correlation length as in the previous analyses. For high values of the initial field we have $-2 \partial \xi / \xi \simeq \partial \bar{\beta}^{2} / \bar{\beta}^{2}$, which leads to the relation $\xi_{0}=\xi \mathcal{L} / a\left(\bar{b}_{0}\right.$ is invariant) under the RG flow. Thus, for any finite $\xi$ during the flow, the bare correlation length $\xi_{0}$ is the system size $L$ or $L_{T}$, corresponding to the quasilong-range order expected of an XY system in one spatial dimension.

Before concluding our RG analysis, we also consider its qualitative predictions for the system magnetization, a measurable quantity. This may be calculated as

$$
M=\frac{\tilde{g} \mu_{B} a}{c} \frac{\partial F}{\partial \bar{\beta}_{0}}=\frac{\tilde{g} \mu_{B} a}{c}\left(\frac{\partial \bar{\beta}}{\partial \bar{\beta}_{0}} \frac{\partial F}{\partial \bar{\beta}}+\frac{\partial g}{\partial \bar{\beta}_{0}} \frac{\partial F}{\partial g}\right) .
$$

Once again, for weak fields we find that $\partial g / \partial \bar{\beta}_{0}=0$ and $\partial \bar{\beta} / \partial \bar{\beta}_{0}=e^{l} \sqrt{1-g_{0} l / 2 \pi}$, where the latter expression will introduce the characteristic length scale $\mathcal{L}$ of the short-range correlated phase. Now for any $F(\bar{b})$ analytic in $\bar{b}$,

$$
M\left(B_{0}\right) \sim \sqrt{1-\frac{g_{0}}{2 \pi} \ln \frac{\mathcal{L}}{a}},
$$

from which it is clear that the magnetization $M\left(B_{0}\right)$ vanishes in the scaling limit $\mathcal{L} \rightarrow \mathcal{L}^{*}$ corresponding to strong coupling (i.e. for all $\bar{b}_{0}$ sufficiently close to the fixed-point 
value $\bar{b}_{0}=0$. Within linear response, this vanishing of the magnetization $M=\chi_{\perp} B$ corresponds to the vanishing of the susceptibility $\chi_{\perp} \sim \ln (\xi / L)$ as the length of the system increases into the disordered state.18 Hence the $\mathrm{RG}$ framework returns a zero magnetization in the regime where quantum fluctuations ensure no spontaneous symmetry breaking. At high fields the magnetization is finite, but its saturation in the physical system limits the useful information which can be deduced from the above approach without inclusion of the saturation constraint (Sec. II).

\section{MAGNETIZATION}

We compute the magnetization from the Lagrangean $\mathcal{L}_{\mathrm{E}}$ of Eq. (2.7), which has separate contributions from in- and out-of-plane spin fluctuations. The calculation is valid in the regime $|b|>\dot{\phi} \equiv \omega$ quoted in Sec. II, and is thus effectively a high-field expansion. $\omega$ denotes the energy of excitations of the system, which may be taken to be spin waves $(\omega=c k)$ in the gapless regime. The magnetization is given by $M=\tilde{g} \mu_{B} \partial F / \partial b$, with $F=-\beta^{-1} \ln Z$ obtained from the partition function $Z=$ $\int D \vartheta D \phi \exp \left(-S_{\mathrm{E}}\right)$. Within the approximations specified below Eq. (2.7), the separable nature of $\mathcal{L}_{\mathrm{E}}$ gives the result $M=M_{o}+M_{i}$. The out-of-plane contribution $M_{o}$ is purely dynamical, in the sense that $\vartheta$ fluctuations appear only in the form $(1 / 2 g) \vartheta G^{-1} \vartheta$. We may write $G^{-1}=$ $G_{0}^{-1}-X$, with the bare propagator given by $G_{0}^{-1}=b^{2}-$ $\partial_{\tau}^{2}-\partial_{x}^{2}$ and the corrections by $X=\dot{\phi}^{2}+2 i b \dot{\phi}$. In the highfield regime $|\dot{\phi} / b|$ is small, so that contributions from $X$ may be neglected. The appropriate piece of the partition function is readily evaluated as

$$
M_{o}=\frac{\beta}{2} \sum_{\mathbf{k}} \frac{1}{\sqrt{b^{2}+c^{2} k^{2}}} \operatorname{coth}\left(\frac{1}{2} \beta \sqrt{b^{2}+c^{2} k^{2}}\right) .
$$

The assumption of an excitation spectrum linear up to the zone boundary is easily altered, for example to a sinusoidal form $\omega_{k}=|\sin k a|$ within the Brillouin zone, which gives rise only to small numerical corrections. Working consistently to lowest order in the small parameter $c / b$, and in limit of low $T$, we find that this contribution is simply a constant, $M_{o}=\frac{1}{2} N_{x} \tilde{g} \mu_{B}$, which corresponds to a spatially uniform state. Small corrections of the form $M_{o}^{\prime} \sim B \ln B$ arise for either type of dispersion.

Treatment of the in-plane variables $\phi$ at finite temperatures is more involved. We first represent the relevant part of the Lagrangean as

$$
\tilde{\mathcal{L}}_{\mathrm{E}}=\frac{1}{2 g}\left[-b^{2}-\phi\left(\partial_{\tau}^{2}+\partial_{x}^{2}\right) \phi+2 i b \dot{\phi}\right]-\frac{i b}{\beta L} \operatorname{Tr} G_{0} \dot{\phi}
$$

to separate the term quadratic in $b$ which gives the classical, linear magnetization. A full treatment of $\phi \in[0,2 \pi]$ involves decompactification to the interval $-\infty<\tilde{\phi}<\infty$ and restoration of periodicity by summation of the partition function over all winding number sectors $m \in \mathcal{Z}$. The phase is represented by $\phi(x, \tau)=\tilde{\phi}(x, \tau)+2 \pi m \tau / \beta$, with $\tilde{\phi}$ a periodic function of the inverse temperature $(\tilde{\phi}(x, \tau+\beta)=\tilde{\phi}(x, \tau))$. This form takes into account the possibility of windings in the space of $\tau$, whereas windings in $x$ may be safely neglected for a large system $\left(L \gg L_{T}\right)$. On expanding the action, noting that all terms $\int_{0}^{\beta} d \tau \dot{\tilde{\phi}}$ vanish (integral of total derivative), and discarding all terms independent of $b$ for the purposes of computing the magnetization, we obtain the corresponding partition function

$$
\tilde{Z}=C_{0} \exp \left\{-\frac{N_{s} b^{2}}{8 \bar{J}}\right\} \sum_{m=-\infty}^{+\infty} \exp \left\{\frac{N_{s} \pi^{2}}{2 \bar{J} \beta} m^{2}+i 2 \pi \alpha m\right\} .
$$

Here $C_{0}$ is a constant and $\alpha=N_{s} b / 4 \bar{J}-N_{x} \ln (\epsilon+$ $\left.\sqrt{1+\epsilon^{2}}\right) / 2 \epsilon$, with $\epsilon=\pi c / a b$, arises from the $\phi$-linear terms in Eq. (6.2). Because $\epsilon$ is a small parameter in the approximation we employ, the second term in $\alpha$ simplifies to $N_{x} / 2$; this is also the origin of the results for $M_{o}$ and $M_{o}^{\prime}$. The sum over $m$ in Eq. (6.3) is simplified on recognizing that $\tilde{Z}$ may be represented by the $\theta_{3}$ function, 31 in terms of which the (logarithmic) $b$-derivative yields

$$
M_{i}=-\tilde{g} \mu_{B}\left\{\frac{N_{s} b}{4 \bar{J}}+\frac{2 \pi N_{s}}{4 \bar{J} \beta} \sum_{m=1}^{\infty}(-1)^{m} \frac{\sin (2 \pi \alpha m)}{\sinh \left(N_{s} \pi^{2} m / 2 \bar{J} \beta\right)}\right\} .
$$

The latter term is a sawtooth form, shown as the quantity $M_{f}$ in Fig. 5(a), which is periodically extended from the interval $-1 / 2<\alpha<1 / 2$ to all of $\alpha$. When superimposed on the linear part contained in the first term of Eq. (6.4), $M_{f}$ gives the step-like form shown in Fig. $5(\mathrm{~b})$. The width of the steps scales as $1 / N_{s}$, so we see that $M_{f}$ is the finite-size correction to the linear magnetization. Such effects are of considerable interest in molecular magnets, where the small number of atoms gives rise to well-defined steps.14 However, $M_{f}$ becomes indiscernible as the system size is taken to the thermodynamic limit, which is the condition we investigate below. In closing this section, we note that evaluation of the magnetization in the NLsM for weak fields $b$, and particularly around $B^{*}$, remains an open problem. Experimental comparison for this regime (Sec. VIII) is facilitated by the knowledge (Secs. III-V) that $M=0$ below $B^{*}$.

\section{SPIN CORRELATION FUNCTIONS}

Correlation functions describing the nature of the spin order may be calculated directly in the NLsM framework from 
(a)

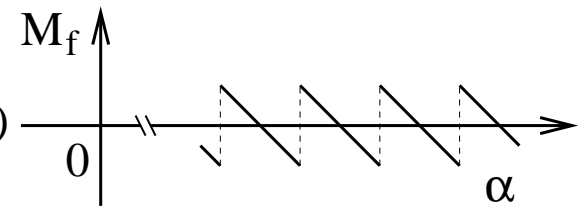

(b)

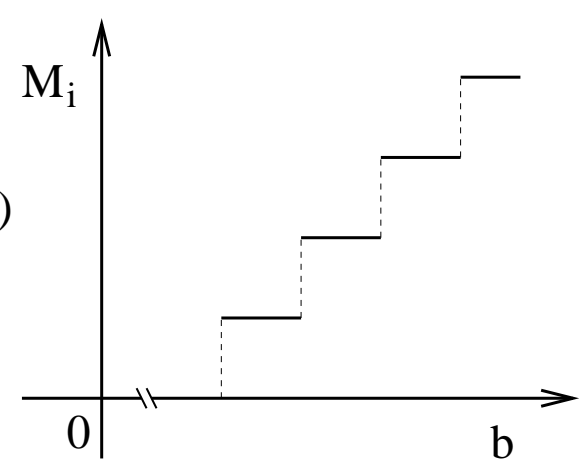

FIG. 5. Schematic representation of finite-size magnetization contribution $M_{i}$ (a) to linear magnetization term (b) at high magnetic fields.

$$
\begin{array}{r}
\left\langle\hat{T}_{\tau} \hat{S}_{i}(x, \tau) \hat{S}_{j}(0,0)\right\rangle=(-1)^{x} S^{2}\left\langle n_{i}(x, \tau) n_{j}(0,0)\right\rangle \\
+(a S)^{2}\left\langle l_{i}(x, \tau) l_{j}(0,0)\right\rangle,
\end{array}
$$

and used to compare the properties of the system at low and high fields. $\hat{T}_{\tau}$ is the ordering operator in imaginary time. In the low-field regime where full symmetry is retained, the gap $\Delta$ to all spin excitations results in exponentially decaying spin correlations, reflecting the short-range nature of the quantum disordered phase.

Above the critical field, as discussed previously, the gap closes and the low-energy features are described by an XY model for the plane normal to the field. The correlation functions in this regime are discussed in detail in App. 2. Because the quantities $\left\langle n_{z}(x, \tau) n_{z}(0,0)\right\rangle$ and $\left\langle l_{+}(x, \tau) l_{-}(0,0)\right\rangle\left(l_{ \pm}\right.$denotes $\left.l_{x} \pm i l_{y}\right)$ depend directly on the gapped, out-of-plane fluctuations in $\vartheta$, these remain short-ranged. However, $\left\langle l_{z}(x, \tau) l_{z}(0,0)\right\rangle$ is proportional to $\partial_{\tau}^{2}\langle\phi(x, \tau) \phi(0,0)\rangle$, which leads to a power-law (or "quasi-long-ranged") decay of the correlation function parallel to the field direction,

$$
\left\langle\hat{T}_{\tau} \hat{S}_{z}(x, \tau) \hat{S}_{z}(0,0)\right\rangle=\frac{\tilde{B}}{8 \pi g}\left[\frac{a^{2}}{(x-i \tau)^{2}}+\frac{a^{2}}{(x+i \tau)^{2}}\right] .
$$

We have omitted here a constant term proportional to the square of the local magnetization. Similarly, $\left\langle n_{+}(x, \tau) n_{-}(0,0)\right\rangle=\langle\exp (i \phi(x, \tau)-i \phi(0,0))\rangle$, which gives

$$
\left\langle\hat{T}_{\tau} \hat{S}_{+}(x, \tau) \hat{S}_{-}(0,0)\right\rangle=\tilde{C}(-1)^{x}\left(\frac{a^{2}}{x^{2}+\tau^{2}}\right)^{(g / 4 \pi)},
$$

a separate power-law form for the correlation function perpendicular to the field direction. $\tilde{B}$ and $\tilde{C}$ are constants. The results for the long-ranged parts of both longitudinal and transverse correlation functions are very similar to those of a Luttinger-liquid description of the gapless, broken-symmetry phase. 32 The correlation exponent in Eq. (7.3) has a direct dependence on the bare coupling constant, and may be identified as an effective Luttinger-liquid exponent

$$
\tilde{K}=\frac{\pi}{2 g}=\frac{\pi S}{2} \sqrt{\frac{J_{x}}{\bar{J}}}=0.42 .
$$

This quantity is independent of the field, and in the NLsM approach is rather close to the constant value 32 $\tilde{K}=1 / 2$ given by bosonization for all ladder systems.

\section{COMPARISON WITH EXPERIMENT}

We turn to a comparison between the preceeding physical ideas and experiments performed on the twochain, $S=1 / 2$ spin ladder material $\mathrm{CuHpCl}$. The magnetization $\mathrm{H}$ illustrates the transition from the gapped regime of unbroken symmetry, and the nature of the broken-symmetry phase, while nuclear magnetic resenance (NMR) measurements of spin relaxation rates 33 reveal some characteristics of the spin correlation functions in both types of ground state.

From Sec. VI, the magnetization takes the simple form

$$
M=-\tilde{g} \mu_{B} N_{s}(b / 4 \bar{J})+M_{o}+O\left(1 / N_{y} S\right),
$$

where $M_{o}$ (Eq. (6.1)) becomes constant for the low temperatures of most interest. While the linear term is always present, we have shown in Secs. IV and V that below a threshold field $B^{*}$, where the system has a spin gap, it is canceled by the corresponding correlation-length term. As discussed in Sec. II, above an upper threshold $\left(B_{c 2}\right)$ the magnetization will saturate at the value $M_{s}$. Here we will for clarity treat saturation as a simple cutoff, rather than pursuing the more complex but fully systematic method of Sec. II, which will be discussed elsewhere. Because the transition at $B_{c 2}$ is from an XY phase with strongly suppressed quantum fluctuations to a fully polarized state with a spin-wave description, an exact treatment is of less interest in the current context. Finally, the $O\left(1 / N_{y} S\right)$ term is included in Eq. (8.1) as a reminder that large corrections are to be expected in any quantitative comparison of a "highly quantum" ( small $N_{y} S$ ) magnetic system with the semiclassical NLsM.

Specializing to the parameters of $\mathrm{CuHpCl}$, the exchange constants deduced from magnetization and susceptibility measurements 5 are $J^{\prime} / k_{B}=13.2 \mathrm{~K}$ and $J / k_{B}$ $=2.4 \mathrm{~K}$, whence $\bar{J} / \mu_{B}=13.3 \mathrm{~T}$ and $J_{x} / \mu_{B}=3.6 \mathrm{~T}$. Taking the simplest case of constant $M_{o}$, and the lower critical field $B_{c 1}$ for onset where $M\left(B_{c 1}\right)=0$, we obtain $B_{c 1}=\bar{J} / \tilde{g} \mu_{B}=6.6 \mathrm{~T}$. The saturation field $B_{c 2}$ is given from $M\left(B_{c 2}\right)=M_{s}$ as $B_{c 2}=\left(4 S / \tilde{g} \mu_{B}\right) \bar{J}=13.3 \mathrm{~T}$. These values agree well with a linear extrapolation of the magnetization data at the lowest temperature in Ref. 5 , which 


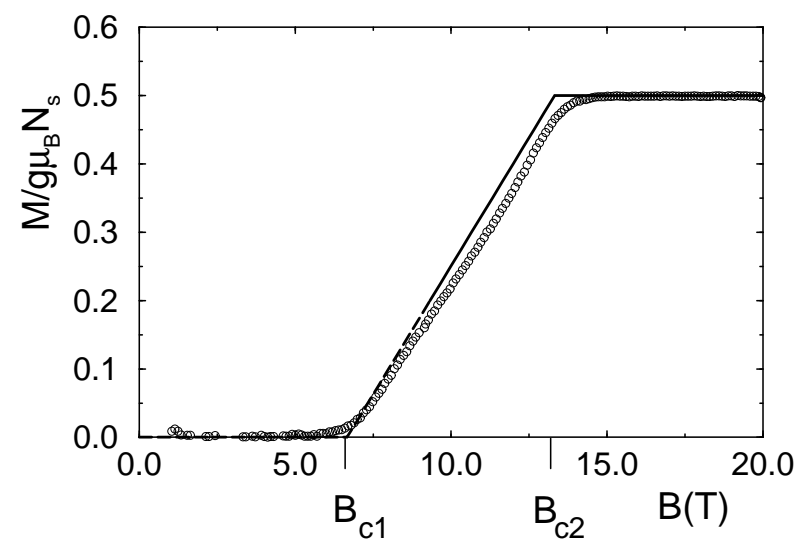

FIG. 6. Computed magnetization for the spin ladder system $\mathrm{CuHpCl}$ (see text). Circles are data from Ref. [5].

yields $B_{c 1}=6.8 \mathrm{~T}$ and $B_{c 2}=13.7 \mathrm{~T}$. The computed magnetization is shown in Fig. 6, where the dashed line indicates the validity limit $(|b| \gtrsim c \pi / a)$ of the calculation.

The NLsM prediction of linear magnetization-appears closer to the data than a repulsive boson model21 34 and other purely 1D approaches, such as bosonization 32 and simulations on a single ladder 34 (or a Haldane chain, 25 which has the same universality class), all of which predict square-root cusps at $B_{c 1}$ and $B_{c 2}$ not evident in the data. $\mathrm{E}$ For a temperature $T=0.42 \mathrm{~K} \sim 25$ this form should be visible in the pure, $1 \mathrm{~d}$ system 34 . This may be indicative of a weak interladder coupling, which also causes the real material to display $3 \mathrm{D}$ order at intermediate $B$ and low $T$. The gradient of the linear region can be written as

$$
\begin{aligned}
\chi_{\mathrm{N} L \sigma \mathrm{M}}^{\perp} & =\frac{1}{N_{s}}\left(\frac{N_{s} S}{4 \bar{J} S-2 \bar{J} / N_{y}}\right) \\
& =\chi_{\mathrm{A} \mathrm{F}}^{\perp}\left(\frac{1}{1-1 /\left(2 N_{y} S\right)}\right),
\end{aligned}
$$

where $\chi_{\mathrm{AF}}^{\perp}=\left(\tilde{g} \mu_{B}\right)^{2} / 4 \bar{J} a^{2}$ is the Néel susceptibility per unit volume, the result for a classical antiferromagnet with effective exchange coupling $\bar{J}$. The last term expresses the deviation from classical behavior as a function of spin magnitude and the number of coupled chains. That the magnetization adopts such a quasi-classical form in the broken-symmetry regime, at least in systems with no topological term, may be ascribed in part to suppression of quantum fluctuations by the field (effective beyond $B_{c 1}$ ), and possibly also to the presence of higher-dimensional couplings.

Further commentary is in order concerning the agreement between the NLsM results and experiment. The semiclassical model has recently been used with considerable success, also for the field response, of small (spatially uniform) systems with rather small $S$. 86 However, the apparent convergence of theory and experiment for the "maximally quantum" case of $N_{y} S=1 \mathrm{in} \mathrm{CuHpCl}$ is in fact a somewhat fortuitous consequence of the param- eter ratio $J / J_{\perp}$. The magnetization computed as above is valid for large $B$, and unlike the correlation length or gap is not sensitive to the coupling constants. Thus the model predictions for the isotropic ladder $\left(J / J^{\prime}=1\right)$, and for the Haldane chain (also $N_{y} S=1$ ), are little different from the above, whereas the known results have much smaller gapped regions. Thus we stress again that the main value of the current approach lies in its qualitative features. From this and other work, 36 it appears that $N_{y} S>3$ is sufficient for the NLsM to provide quantitative accuracy, although the gap is then very small.

The experimental comparison gives additional perspective on the utility of the spin stiffness and $1 / N$ treatments (Secs. III and IV). We deduce from $L_{m}^{*}$ that $B^{*}=0.32 \bar{J} / \tilde{g} \mu_{B}=4.2 \mathrm{~T}$, a value rather lower than $B_{c 1}$ above, and emphasize that in the regime between $B^{*}$ and $B_{c 1}$ neither treatment is reliable. Following the RG analysis, the field scale $\bar{b}_{*}$ from the numerical solution gives $B_{*}=1.6 \mathrm{~T}$, although prefactor corrections (above) can be expected.

The other category of experiments performed on $\mathrm{CuH}-$ $\mathrm{pCl}$ is the measurement of NMR spin relaxation rates, which probe the spin-spin correlation functions discussed in Sec. VII. The experimental observable is the spinlattice relaxation rate, given in $d$ dimensions by

$$
\frac{1}{\mathrm{~T}_{1}}=\lim _{\omega \rightarrow 0} \frac{2 k_{\mathrm{B}} T}{\hbar \omega} \sum_{i j} \int \frac{d^{d} q}{(2 \pi)^{d}} F_{i j}(\mathbf{q}) \chi_{i j}(\mathbf{q}, \omega, T),
$$

in which $F_{i j}$ are hyperfine coupling constants. This is obtained from the dynamic susceptibility

$$
\begin{aligned}
\chi_{i j}(\mathbf{q}, \omega, T)= & -i \int d t d \mathbf{x} e^{i \omega t+i \mathbf{q} \cdot \mathbf{x}} \\
& \times \Theta(t)\left\langle\left[\hat{S}_{i}(\mathbf{x}, t), \hat{S}_{j}(\mathbf{0}, 0)\right]\right\rangle_{T},
\end{aligned}
$$

which in turn makes contact with the results of Sec. VII. By straightforward scaling, spin-spin correlation functions $\left\langle\hat{T}_{\tau} \hat{S}_{i}(x, \tau) \hat{S}_{j}(0,0)\right\rangle$ decaying exponentially in $1+1 \mathrm{~d}$ space correspond to relaxation rates which are exponentially activated in temperature, for $T$ below the characteristic energy scale $A$. This is observed in experiments for fields $B<B_{c 1} .33$ Similarly, power-law spatial decays correspond to thermal power laws in relaxation rates: by performing separate Fourier transformations on $x-i \tau$ and $x+i \tau$ and extracting the energy-dependence in the long-wavelength limit $(k \rightarrow 0)$, if

$$
\left\langle\hat{T}_{\tau} \hat{S}_{i}(x, \tau) \hat{S}_{j}(0,0)\right\rangle \propto \delta_{i j}\left(x^{2}+\tau^{2}\right)^{-\alpha}
$$

then

$$
1 / \mathrm{T}_{1} \propto T^{(2 \alpha-2)+1},
$$

whence from Eqs. $(7.2,7.3)$ the relaxation rate for the ladder system is

$$
\left.\frac{1}{\mathrm{~T}_{1}}\right|_{\text {ladder }}=A_{\|} T+B_{\perp} T^{-g / 2 \pi-1} .
$$


$A_{\|}$and $B_{\perp}$ are constants related to the hyperfine coupling. An identical result was obtained in the LuttingerLiquid formulation of Ref. 32. In addition to the activated form in the gapped regimes at $B<B_{1}$ and $B>$ $B_{c 2}$, the experimental measurements of $1 / T_{1} 33$ show clear power-law divergence in the intermediate field regime, fully consistent with expectations (Sec. VII) based on a model of symmetry breaking by the effect of the field on quantum fluctuations. However, because the data 33 cover at most one decade in temperature, while typically 3-4 decades are required to identify the exponent within meaningful bounds, we may not attempt to make any quantitative deductions (Eq. (7.4) ) concerning the coupling constant $g$.

\section{SUMMARY}

In conclusion, the nonlinear $\sigma$ model description of quantum antiferromagnets in an external field provides a versatile framework for illustrating the breaking and restoration of symmetries. The case of $O(3)$ spins has a variety of physical realizations, which illustrate clearly the transition from the gapped, short-range ordered phase where full spin symmetry is maintained by quantum fluctuations, to a gapless, quasi-long-range ordered regime where the $O(3)$ symmetry is reduced to $O(2)$ by the field.

The foregoing analysis is not restricted to $\mathrm{CuHpCl}$, but applies to any system with a trivial topological term $\sum_{i} P_{i}$ (Eq. (2.3)). We comment here on its application to materials in two other classes. CFTD is a planar $S=\frac{1}{2}$ Heisenberg AF, of a type studied extensively in connection with the high-temperature superconductivity problem 1 and known 9 to have $\mathrm{AF}$ order at $T=0$. In an applied field one expects linear magnetization characteristics with the Néel susceptibility, beginning at $B=0$, but not saturating because the superexchange interaction $J=9.4 \mathrm{meV}$ is beyond the reach of laboratory fields. Magnetization results on further recently-synthesized, planar Cysompounds with significantly smaller exchange constants37 may show evidence of a logarithmic approach to saturation, as expected from spin-wave theory for a purely $2 \mathrm{~d}$ system, and obtainable in the NLsM by inclusion of the total spin contraint as in Sec. II. The cases of most interest are those exhibiting the physics of the Haldane gap. The primary example is the $S=1 \mathrm{AF}$ chain, of which NENP is considered a prototypical case (but for the complication of a large single-ion anisotropy). The present study yields the same qualitative features of a gapped regime with zero magnetization, followed by approximately linear behavior towards saturation (which could not be achieved), as in experiment.3 Quantitative accord with experimental transition fields, and numerical simulations, 35 does not match that of Sec. VIII for $\mathrm{CuH}-$ pCl. Finally, there has been considerable recent interest in the possibility of field-induced magnetization plateaus in certain systems: in a NLsM approach these may be expected, for example in $S>1$ chains, when the field strength is such that the projected in-plane spin $\left|S \mathbf{n}_{\perp}\right|$ is of integer amplitude, leading to a gapped phase.

\section{ACKNOWLEDGMENTS}

We are grateful to G. Chaboussant, F. Mila, and S. Sachdev for useful discussions, and thank the Swiss National Fund for financial support. BN acknowledges the generosity of the Treubelfonds.

\section{APPENDIX A: RENORMALIZATION GROUP EQUATIONS}

The appendix presents the derivation of the integral forms (5.3,5.4) of the RG equations for the coupling constant and field. We begin with the Lagrangean for the NLsM in a magnetic field in the form of Eq. (5.1),

$$
\mathcal{L}_{E}=\frac{1}{2 g}\left[\left(\partial_{\mu} \mathbf{n}\right)^{2}-\bar{b}^{2}\left(1-n_{z}^{2}\right)+4 i \bar{b} n_{x} \dot{n}_{y}\right],
$$

where the parameters are defined in Secs. II and III, and the last term is obtained by partial integration of one component of the cross product. The staggered magnetization satisfies $|\mathbf{n}|^{2}=1$ and (Sec. II) lies predominantly in the $(x, y)$ plane, so that we may choose $\left|n_{z}\right| \ll 1$ and specify the components of $\mathbf{n}$ by $\left(n_{x}=\sqrt{1-n_{z}^{2}} \cos \phi\right.$, $\left.n_{y}=\sqrt{1-n_{z}^{2}} \sin \phi, n_{z}\right)$, with $0 \leq \phi<2 \pi$. Using these variables, the cross product term gives $n_{x} \dot{n}_{y}-n_{y} \dot{n}_{x}=$ $\left(1-n_{z}^{2}\right) \dot{\phi}$, and the gradient term becomes

$$
\left(\partial_{\mu} \mathbf{n}\right)^{2}=\left(\partial_{\mu} n_{z}\right)^{2}+\left(1-n_{z}^{2}\right)\left(\partial_{\mu} \phi\right)^{2}+\frac{\left(n_{z} \partial_{\mu} n_{z}\right)^{2}}{1-n_{z}^{2}} .
$$

On making the substitution $n_{z}=\sqrt{g} \sigma_{z}$, where the coupling constant $g$ is taken to be a small parameter (weakcoupling expansion), the Lagrangean (A1) is exactly represented as

$$
\begin{gathered}
\mathcal{L}_{E}=\frac{1}{2 g}\left(1-g \sigma_{z}^{2}\right)\left[\left(\partial_{\mu} \phi\right)^{2}+2 i \bar{b} \dot{\phi}-\bar{b}^{2}\right]+\frac{1}{2}\left(\partial_{\mu} \sigma_{z}\right)^{2} \\
+\frac{1}{2} \frac{g}{\left(1-g \sigma_{z}^{2}\right)}\left(\sigma_{z} \partial_{\mu} \sigma_{z}\right)^{2} .
\end{gathered}
$$

With the definition $A^{\prime}=\left(\partial_{\mu} \phi\right)^{2}+2 i \bar{b} \dot{\phi}, \mathcal{L}_{E}$ may be expanded in the power series in $g$

$$
\begin{aligned}
\mathcal{L}_{E}=\frac{1}{2 g}\left[A^{\prime}-\bar{b}^{2}\right]+\frac{1}{2}\left(\partial_{\mu} \sigma_{z}\right)^{2}-\frac{1}{2}\left(\sigma_{z}\right)^{2}\left[A^{\prime}-\bar{b}^{2}\right] & \\
& -\frac{1}{2} g\left(\sigma_{z} \partial_{\mu} \sigma_{z}\right)^{2}+\frac{1}{2} g^{2} \sigma_{z}^{2}\left(\sigma_{z} \partial_{\mu} \sigma_{z}\right)^{2}+O\left(g^{3}\right) .
\end{aligned}
$$

We proceed to one-loop order by neglecting those terms $O(g)$ and higher, and by following the Wilson RG technique of integrating out states of higher momenta around a finite upper cutoff. 38.22 . 13 In this process the renormalizing effect of the fast Fourier modes of $\sigma_{z}$ is taken into 
account, whereas the slow variable $\phi(\tau, x)$ and its (small) derivative terms give no contribution at high momenta. We compute the quantity

$I=\int \mathcal{D} \sigma_{z}(\mathbf{k}) e^{-\frac{1}{2} \int d \mathbf{x}\left[\frac{1}{2 g}\left(A^{\prime}-\bar{b}^{2}\right)+\sigma_{z}\left(-\partial_{\mu}^{2}-A^{\prime}+\bar{b}^{2}\right) \sigma_{z}+O(g)\right]}$

in the isotropic momentum shell $\gamma \Lambda<|\mathbf{k}|<\Lambda$, where $\gamma<1$ and $\Lambda=\pi / a$. Evaluation of the Gaussian integral yields

$$
\begin{aligned}
I & =\prod_{\gamma \Lambda<|\mathbf{k}|<\Lambda} \sqrt{\frac{2 \pi}{\mathbf{k}^{2}-A^{\prime}+\bar{b}^{2}}} e^{-\frac{1}{2 g}\left[A^{\prime}-\bar{b}^{2}\right]} \\
& =e^{-\frac{1}{2} \operatorname{Tr}^{\prime} \ln \left(-\partial_{\mu}^{2}-A^{\prime}+\bar{b}^{2}\right)} e^{-\frac{1}{2 g}\left[A^{\prime}-\bar{b}^{2}\right]}
\end{aligned}
$$

in which the small quantity $A^{\prime}$ permits the expansions

$$
\begin{aligned}
\operatorname{Tr}^{\prime} \ln \left(-\partial_{\mu}^{2}-A^{\prime}+\bar{b}^{2}\right)= & \operatorname{Tr}^{\prime} \ln \left(-\partial_{\mu}^{2}+\bar{b}^{2}\right) \\
& -\operatorname{Tr}^{\prime}\left[\frac{1}{-\partial_{\mu}^{2}+\bar{b}^{2}} A^{\prime}\right]+O\left(A^{\prime}\right)^{2}
\end{aligned}
$$

and

$$
\operatorname{Tr}^{\prime}\left[\frac{1}{-\partial_{\mu}^{2}+\bar{b}^{2}} A^{\prime}\right] \simeq \operatorname{Tr}^{\prime}\left[\frac{1}{-\partial_{\mu}^{2}+\bar{b}^{2}}\right] A^{\prime}
$$

Substituting these results into the original Lagrangean $\mathcal{L}_{E}$ (A4) returns the new, effective Lagrangean on renormalized lattice length scale $a^{\prime}$,

$$
\begin{aligned}
\mathcal{L}_{\text {eff }}=\frac{1}{2} & \left(\frac{1}{g_{0}}-\operatorname{Tr}^{\prime} \frac{1}{-\partial_{\mu}^{2}+\bar{b}^{2}}\right) A^{\prime} \\
& +\frac{1}{2}\left(\partial_{\mu} \sigma_{z}\right)^{2}-\frac{1}{2}\left(\sigma_{z}\right)^{2}\left[A^{\prime}-\bar{b}^{2}\right] \\
& -\frac{1}{2}\left(\frac{1}{g_{0}} \bar{b}^{2}-\operatorname{Tr}^{\prime} \ln \left(-\partial_{\mu}^{2}+\bar{b}^{2}\right)\right) .
\end{aligned}
$$

Finally, by comparison of $\mathcal{L}_{\text {eff }}$ with $\mathcal{L}_{E}$, we may identify the renormalized coupling and magnetic field terms as expressed in Eqs. (5.3,5.4).

\section{APPENDIX B: SPIN CORRELATION FUNCTIONS}

In this appendix we present the calculation of spin-spin correlation functions, in real space and inverse temperature, for the gapless, high-field phase of the NLsM. The latter part of Sec. VII illustrates the transformation of these quantities for comparison with experiment. It is apparent from Eq. (7.1) that four such functions must be considered, corresponding to correlations of the staggered and uniform spin components in directions parallel $(i, j=z)$ and perpendicular $(i, j= \pm)$ to the field. We reexpress the spin components $\mathbf{n}$ and $\mathbf{l}$ in terms of the angles $\vartheta$ and $\phi$ (Sec. II), for which the propagators are known.

The parallel response function for the staggered spin components is computed using

$$
\left\langle n_{z}(\mathbf{x}) n_{z}(\mathbf{0})\right\rangle=\int \mathcal{D} \mathbf{n} n_{z}(\mathbf{x}) n_{z}(\mathbf{0}) e^{-\mathcal{S}[\mathbf{n}]},
$$

where $n_{z}=\cos \theta=\sin \vartheta \simeq \vartheta$ for small angular deviations from the plane determined by the field. One expects the linear expansion to be valid for the lowest-order form of the correlation function, and may use it also in the approximation $\int \mathcal{D}(\cos \vartheta) \simeq \int \mathcal{D} \vartheta$. Now

$$
\begin{aligned}
& \left\langle n_{z}(x, \tau) n_{z}(0,0)\right\rangle=\int \mathcal{D} \vartheta \vartheta(\mathbf{x}) \vartheta(\mathbf{0}) \exp \left\{-\frac{1}{2 g} \vartheta \cdot G_{0}^{-1} \vartheta\right\} \\
& =\lim _{j \rightarrow 0} \frac{\delta}{\delta j_{x}} \frac{\delta}{\delta j_{0}} \int \mathcal{D} \vartheta \exp \left\{-\frac{1}{2 g} \vartheta \cdot G_{0}^{-1} \vartheta+j \cdot \vartheta\right\},
\end{aligned}
$$

in which $\vartheta$ denotes a vector representing states at all $\mathbf{x}=(\tau, x), G_{0}^{-1}=-\partial_{\mu}^{2}+\bar{b}^{2}$ is the matrix propagator for modes of $\vartheta$, and the current $j(\mathbf{x})$ is taken to zero at the end of the calculation. Evaluation of the Gaussian integral yields

$$
\lim _{j \rightarrow 0} \frac{\delta}{\delta j_{x}} \frac{\delta}{\delta j_{0}} \exp \left\{\frac{1}{2} g j \cdot G_{0} j\right\}=g G_{0}(\mathbf{x}, \mathbf{0}),
$$

from which the propagator component is given by resolution into plane wave states according to

$$
G_{0}(\mathbf{x}, \mathbf{0})=\left\langle\mathbf{x}\left|\frac{1}{-\partial_{\mu}^{2}+\bar{b}^{2}}\right| \mathbf{0}\right\rangle=\frac{1}{L L_{T}} \sum_{\mathbf{k}} \frac{e^{i \mathbf{k} \cdot \mathbf{x}}}{\mathbf{k}^{2}+\bar{b}^{2}},
$$

where $\mathbf{k}=\left(\omega_{n}, k\right)$. For the interesting limit of large distance $\left(\bar{b} \sqrt{x^{2}+\tau^{2}}>1\right)$, we conclude that the correlation function

$$
\left\langle n_{z}(x, \tau) n_{z}(0,0)\right\rangle=\frac{\tilde{A}}{\sqrt{\bar{b} \sqrt{x^{2}+\tau^{2}}}} e^{-\bar{b} \sqrt{x^{2}+\tau^{2}}},
$$

where $\tilde{A}$ is a constant, has the exponentially decaying form in space and inverse temperature expected from its origin in the gapped, out-of-plane excitations. is 18

$$
\begin{aligned}
\left\langle l_{z}(\mathbf{x}) l_{z}(\mathbf{0})\right\rangle= & \left\langle l_{z}^{\mathrm{cl}}(\mathbf{z}) l_{-}^{\mathrm{cl}}(\mathbf{0})\right\rangle \\
& +\frac{g}{4 c} \frac{1}{L L_{T}} \sum_{\mathbf{q}} e^{i \mathbf{q} \cdot \mathbf{x}}\left(1-\left\langle n_{z}(\mathbf{x}) n_{z}(\mathbf{0})\right\rangle\right) .
\end{aligned}
$$

The latter term is a quantum correction arising from the constraint $\mathbf{l} \cdot \mathbf{n}=0$, which is unaffected by the field, and has the value $(g / 4 c)\left(\delta_{\mathbf{x}, \mathbf{0}}-\left\langle\left(n_{z}(\mathbf{0})\right)^{2}\right\rangle\right)$, i.e. is a local quantity which contributes only to a constant proportional to the square of the local magnetization. From Eq. (2.4), the expression for $l_{z}^{\mathrm{cl}}$ may be simplified by noting that the field term $\mathbf{b}=\left(0,0, b_{z}\right)$ selects 
$n_{z}$, which in turn introduces $\vartheta$, and thus only exponentially decaying contributions. The remaining term is $(\mathbf{n} \wedge \dot{\mathbf{n}})_{z}=\dot{\phi} \sin ^{2} \vartheta \simeq \dot{\phi}$ only. With the exception of further constant terms $\left(\left\langle n_{z}^{2}(\mathbf{0})\right\rangle\right)$, which are neglected both here and in Eq. (7.2), we may restrict our considerations to

$$
\begin{aligned}
(a S)^{2}\left\langle l_{z}(\mathbf{x}) l_{z}(\mathbf{0})\right\rangle & =-\frac{1}{(4 \bar{J})^{2}}\langle\dot{\phi}(\mathbf{x}) \dot{\phi}(\mathbf{0})\rangle \\
& =\frac{1}{(4 \bar{J})^{2}} \partial_{\tau}^{2}\langle\phi(\mathbf{x}) \phi(\mathbf{0})\rangle
\end{aligned}
$$

We see now that we are dealing only with the gapless, inplane modes, which can be expected to give a power-law correlation function at long distances, and the treatment is identical to the Luttinger liquid. By the same technique as above (Eqs. (B2 B3)), the correlation function for the azimuthal angles is given by

$$
\langle\phi(\mathbf{x}) \phi(\mathbf{0})\rangle=2 g \tilde{B} \tilde{G}_{0}(\mathbf{x}, \mathbf{0}),
$$

with propagator $\tilde{G}_{0}=-\partial_{\mu}^{2}$ for in-plane modes, and $\tilde{B}$ a constant. Use of the result (expressed in rectangular coordinates with $c$ retained explicitly)

$$
\begin{gathered}
\frac{c}{L L_{T}} \sum_{\omega, k \neq 0} \frac{\omega^{2}}{k^{2}+(\omega / c)^{2}} e^{i k x+i \omega \tau}=\frac{c^{3}}{2 \pi} \frac{x^{2}-c^{2} \tau^{2}}{\left[x^{2}+c^{2} \tau^{2}\right]^{2}} \\
=\frac{c^{3}}{4 \pi}\left[\frac{1}{(x-i c \tau)^{2}}+\frac{1}{(x+i c \tau)^{2}}\right]
\end{gathered}
$$

leads directly to Eq. (7.2). The prefactor is obtained from the identity $c / 2 \bar{J}=a / g$, while further identification of $\pi / 2 g$ with $\tilde{K}(7.4)$ yields the result of Ref. 32 . Because we are not interested in finite-size properties for discussing the asymptotic behavior of correlation functions, we ignore the zero modes (Eq. (B9)) occurring in the Luttinger liquid. Their presence is connected with decompactification of $\phi$ and the associated winding number (Sec. VI), and their treatment discussed in Ref. 39 .

Moving to the correlation functions normal to the applied field, that for the staggered spin components has the simple form

$$
\Xi=\left\langle n_{+}(\mathbf{x}) n_{-}(\mathbf{0})\right\rangle=\langle\exp (i \phi(\mathbf{x})-i \phi(\mathbf{0}))\rangle .
$$

Defining the function $j\left(\mathbf{x}^{\prime}\right)=\delta\left(\mathbf{x}-\mathbf{x}^{\prime}\right)-\delta\left(\mathbf{x}^{\prime}\right)$, and using the equalities $\tilde{G}_{0}(\mathbf{x}, \mathbf{x})=\tilde{G}_{0}(\mathbf{0}, \mathbf{0})$ and $\tilde{G}_{0}(\mathbf{x}, \mathbf{0})=$ $\tilde{G}_{0}(\mathbf{0}, \mathbf{x})$, permits one to write

$$
\begin{aligned}
\Xi & =\int \mathcal{D} \phi \exp \left\{-\frac{1}{2 g} \phi \cdot \tilde{G}_{0}^{-1} \phi+i j \cdot \phi\right\} \\
& =\left(\operatorname{Det} \tilde{G}_{0}^{-1}\right)^{-1 / 2} \exp \left\{-\frac{1}{2} g j \cdot \tilde{G}_{0} j\right\} \\
& =C^{\prime} \exp \left\{-g\left[\tilde{G}_{0}(\mathbf{0}, \mathbf{0})-\tilde{G}_{0}(\mathbf{x}, \mathbf{0})\right]\right\}
\end{aligned}
$$

where $C^{\prime}$ is a constant. The propagators are evaluated as above, to give

$$
\tilde{G}_{0}(\mathbf{0}, \mathbf{0})=\frac{1}{L L_{T}} \sum_{\mathbf{k} \neq \mathbf{0}} \frac{1}{\mathbf{k}^{2}}=\frac{1}{2 \pi} \ln \left(\frac{\mathcal{L}}{a}\right)
$$

and

$$
\tilde{G}_{0}(\mathbf{x}, \mathbf{0})=\frac{1}{L L_{T}} \sum_{\mathbf{k} \neq \mathbf{0}} \frac{e^{i \mathbf{k} \cdot \mathbf{x}}}{\mathbf{k}^{2}}=-\frac{1}{4 \pi} \ln \left(\frac{(2 \pi)^{2}\left(x^{2}+\tau^{2}\right)}{\mathcal{L}^{2}}\right)
$$

both of which are cut off by the system length scale (Sec. V) $\mathcal{L}=\min \left[L, L_{T}\right]$. Finally,

$$
\left\langle n_{+}(\mathbf{x}) n_{-}(\mathbf{0})\right\rangle=\tilde{C}\left(\frac{\mathcal{L}}{a}\right)^{-\frac{g}{2 \pi}}\left(\frac{x^{2}+\tau^{2}}{\mathcal{L}^{2}}\right)^{-\frac{g}{4 \pi}}
$$

for altered constant $\tilde{C}$, returns the result in Eq. (7.3). That this is the only power-law contribution to $\left\langle S_{+}(\mathbf{x}) S_{-}(\mathbf{0})\right\rangle$ is easily seen from

$$
\begin{aligned}
\left\langle l_{+}^{\mathrm{cl}}(\mathbf{x}) l_{-}^{\mathrm{cl}}(\mathbf{0})\right\rangle & =\left\langle e^{i \phi(\mathbf{x})-i \phi(\mathbf{0})} \dot{\vartheta}(\mathbf{x}) \dot{\vartheta}(\mathbf{0})\right\rangle \\
& =-\left\langle e^{i \phi(\mathbf{x})-i \phi(\mathbf{0})}\right\rangle\left[\partial_{\tau}^{2}\langle\vartheta(\mathbf{x}) \vartheta(\mathbf{0})\rangle\right]
\end{aligned}
$$

whose dependence on $\vartheta$ guarantees an exponential decay of correlations.

${ }^{1}$ F. D. M. Haldane, Phys. Lett. 93A, 464 (1983); Phys. Rev. Lett. 50, 1153 (1983).

${ }^{2}$ M. Oshikawa, M. Yamanaka, and I. Affleck, Phys. Rev. Lett. 78, 1984 (1997).

${ }^{3}$ Y. Ajiro, T. Goto, H. Kikuchi, T. Sakakibara, and T. Inami, Phys. Rev. Lett. 63, 1424 (1989).

${ }^{4}$ A. Harrison, S. J. Clarke, T. E. Mason, G. J. McIntyre, and D. Visser, J. Magn. Magn. Mater. 104-107, 557 (1992).

${ }^{5}$ G. Chaboussant, P. A. Crowell, L. P. Lévy, O. Piovesana, A. Madouri, and D. Mailly, Phys. Rev. B 55, 3046 (1997).

${ }^{6}$ P. R. Hammar, D. H. Reich, C. Broholm, and F. Trouw, Phys. Rev. B 57, 7846 (1998).

${ }^{7}$ I. Affleck, J. Phys.: Condens. Matter 1, 3047 (1989).

${ }^{8}$ S. Chakravarty, B. I. Halperin, and D. R. Nelson, Phys. Rev. B 39, 2344 (1989).

${ }^{9}$ see E. Manousakis, Rev. Mod. Phys. 63, 1 (1991).

${ }^{10}$ A. M. Polyakov, Gauge Fields and Strings (Harwood, London, 1986).

${ }^{11}$ H. J. Mikeska, J. Phys. C 13, 2913 (1980); A. F. Andreev and V. I. Marchenko, Sov. Phys. Usp. 23, 21 (1980).

${ }^{12}$ E. Fradkin, Field Theories of Condensed Matter Systems (Addison-Wesley, Redwood City, 1991).

13 A. Auerbach, Interacting Electrons and Quantum Magnetism (Springer, New York, 1994).

${ }^{14}$ D. Loss, in Dynamical Properties of Unconventional Magnetic Systems, edited by A.T. Skjeltorp and D. Sherrington (Kluwer Academic Publishers, Dordrecht, 1998). 
${ }^{15}$ We have made the assumption, well justified for the parameters of $\mathrm{CuHpCl}$ (Sec. VIII), that all relevant energies should be below the finite-size gap, $E_{0}=2 \pi S J_{y}$, of spin waves transverse to the ladder.

${ }^{16}$ D. V. Kveshchenko, Phys. Rev. B 50, 380 (1994).

${ }^{17}$ G. Sierra, J. Math. Phys. A 29, 3289 (1996).

18 S. Allen and D. Loss, Physica A, 239, 47 (1997).

19 D. Loss and D. L. Maslov, Phys. Rev. Lett. 74, 178 (1995).

${ }^{20}$ For classical systems see also J. Zinn-Justin, Quantum Field Theory and Critical Phenomena (Oxford Univ. Press, New York, 1993), 2nd ed.

${ }^{21}$ Before saturation there is no true long-range order, but the field imposes a quasi-long-range order of the $X Y$-model type: I. Affleck, Phys. Rev. B 43, 3215 (1991).

${ }^{22}$ D. S. Fisher, Phys. Rev. B 39, 11783 (1989).

${ }^{23}$ S. Sachdev, Z. Phys. B 94, 469 (1994).

${ }^{24}$ In the Fermi liquid formalism 26 the field is absorbed in an effectire chemical potential. As deduced from Seitz' theorem, 27 the chemical potential at fixed temperature is renormalized by interactions, and not necessarily weakly, although the Fermi wave vector $k_{F}$ is not renormalized according to Luttinger's theorem. Similarly, in a Luttinger liquid the Zeeman interaction is in general also renormalized: 28 in the $\mathrm{RG}$ treatment of this case, the Luttinger liquid parameters $\left(K_{\sigma}\right)$ themselves becomplogarithmically dependent on the magnetic field alone2s (cf. Eq. (5.7)). Neither of these results satisfies the scaling hypothesis of Ref. 23.

${ }^{25}$ We note that the gauge theory (Eq. (5.8) ) is non-Abelian, 23 an important consequence of which is that different field components need not renormalize in the same way. Thus the result of Eq. (5.7) does not conflicit with the discussion of gauge invariance and RG methods introducing finite momentum cutoffs in Refs. 29, 30, which concerns the Abelian case.

${ }^{26}$ D. Pines and P. Nozières, Theory of Quantum Liquids (Addison-Wesley, Redwood City, 1966).

27 G. D. Mahan, Many-Particle Physics (Plenum, New York, 1990), 2nd ed.

${ }^{28}$ A. O. Gogolin, A. A. Nersesyan, and A. M. Tsvelik, Bosonization Approach to Strongly Correlated Systems (Cambridge University Press, Cambridge, 1998).

${ }^{29}$ B. I. Halperin, T. C. Lubensky, and S.-K. Ma, Phys. Rev. Lett. 32, 292 (1974).

${ }^{30}$ J.-H. Chen, T. C. Lubensky, and D. R. Nelson, Phys. Rev. B 17, 4274 (1978).

${ }^{31}$ M. Abramovitz and I. A. Stegun, Handbook of Mathematical Functions (Dover, New York, 1972).

${ }^{32}$ R. Chitra and T. Giamarchi, Phys. Rev. B 55, 5819 (1997).

${ }^{33}$ G.Chaboussant, Y. Fagot-Revurat, M.-H. Julien, M. E. Hanson, C. Berthier, M. Horvatic, L. P. Lévy and O. Piovesana, Phys. Rev. Lett. 80, 2713 (1998).

${ }^{34}$ C. A. Hayward, D. Poilblanc, and L. P. Lévy, Phys. Rev. B 54, R12649 (1996).

${ }^{35}$ V. A. Kashurnikov, N. V. Prokof'ev, B. V. Svistunov, and M. Troyer, Phys. Rev. B 59, 1162 (1999).

${ }^{36}$ A. Chiolero and D. Loss, Phys. Rev. Lett. 80, 169 (1998).

${ }^{37}$ A. S. Albrecht, C. M. Wynn, C. P. Landee, and M. M. Turnbull, unpublished.

38 J. B. Kogut, Rev. Mod. Phys. 51, 659 (1979).
39 D. Loss, Phys. Rev. Lett. 69, 343 (1992). 